Ann. Zootech., I969, 18 (3), 317-329.

\title{
INFLUENCE DE L'INTERVALLE ENTRE LA PARTURITION ET LA SAILLIE SUR LA FERTILITÉ ET LA PROLIFICITÉ DES LAPINS
}

\author{
M. PRUD'HON, R. ROUVIER, Jocelyne CAEL (*), et L. BEL \\ Station de physiologie animale, \\ Centre de Recherches agronomiques du Midi, \\ École nationale supérieure agronomique, 34 - Montpellier \\ Station de Génétique quantitative et appliquée, \\ Centre national de Recherches zootechniques, 78 -Jouy-en-Josas. \\ Institut national de la Recherche agronomique.
}

\section{SOMMAIRE}

Deux essais ont été réalisés, dans deux élevages distincts, dans le but d'étudier l'influence de l'intervalle entre la parturition et la saillie sur la fertilité et la prolificité des lapines.

L'essai I concerne des lapines "communes " et l'essai 2 des lapines, pour la plupart, de race Argenté de Champagne. Les données ont été divisées en trois lots dans l'essai $\mathbf{r}$, en 4 lots dans l'essai 2 , en fonction de l'intervalle entre la parturition et la saillie suivante, les valeurs de ces intervalles étant respectivement:
Essai I :
o à 6 jours (lot $\mathrm{I})$
Io à zo jours (lot 2$)$
plus de 30 jours (lot 3 )
Essai 2 :
I à 7 jours (lot $\mathrm{x}$ )
9 à 17 jours $(\operatorname{lot} 2)$
20 à 27 jours (lot 3$)$
plus de 30 jours (lot 4 )

Les taux de gestation (nombre de mises-bas pour Ioo saillies) sont significativement plus faibles dans les lots I des deux essais que dans les lots 2 (respectivement 56 et 45 p. Ioo contre 85 et 82 p. Ioo).

Dans l'essai I, les nombres moyens de nés " total » et nés "vivants " par portée sont significativement plus faibles dans le lot I que dans les autres lots, la différence étant de l'ordre de I à I, 5 lapereau. La même tendance est observée dans l'essai 2 , mais dans ce cas la différence n'est pas significative.

L'influence du nombre de lapereaux nés et allaités jusqu'à I 4 jours à une portée sur les résultats de la saillie suivante (taux de gestation, taux de prolificité) a aussi été examinée. Cette étude n'a pas mis en évidence d'antagonisme entre gestation et allaitement, dans les conditions d'alimentation de nos animaux, lorsque la saillie fécondante a lieu to à 20 jours après la parturition.

Ces résultats ne permettent pas de préciser le rythme optimum de reproduction des lapines mais indiquent qu'il est possible d'obtenir des résultats zootechniques satisfaisants en faisant saillir les lapines dès la parturition et en accouplant à nouveau les lapines non gestantes à partir du ro jour après cette saillie.

* Une partie des résultats présentés ici a fait l'objet d'un mémoire de fin d'études présenté par Jocelyne Sinse-CAEL à l'École nationale des Ingénieurs de Travaux agricoles, - Bordeaux.

Innales de Zootechnie. - r969. 


\section{INTRODUCTION}

Les qualités maternelles des lapines et leurs aptitudes reproductrices sont également remarquables. Traditionnellement on a surtout exploité les premières en laissant profiter les lapereaux de l'allaitement maternel jusqu'à 1'âge de 6 à 8 semaines. Récemment, la mise au point de techniques de sevrage précoce et d'alimentation des lapereaux par voie sèche dès l'âge de 3 semaines (DON GUTHRIE, I966), ou 2 semaines (PRUD'HON et BEL, I968) a conduit à rechercher les possibilités d'utilisation intensive du potentiel de reproduction de ces femelles. Cette recherche peut s'envisager dans le cadre d'un âge au sevrage pouvant aller jusqu'à 28 jours, la durée de gestation de la lapine étant de l'ordre de 3 I à 32 jours. La mise au point d'un rythme de reproduction avec intervalles entre parturitions successives aussi courts que possible présente un grand intérêt aussi bien pour le producteur de lapins de boucherie que pour le sélectionneur. D'une part, cela permettrait d'accroître, spectaculairement semble-t-il, le nombre de lapins sevrés par lapine et par an; d'autre part, cela permettrait d'améliorer la pression de sélection et de juger plus rapidement les aptitudes des animaux.

On sait depuis longtemps (Hammond et Marshai, I, I925) que très peu de temps après la parturition, les lapines acceptent l'accouplement, ovulent et peuvent être gestantes. CASADY (I965) a montré, sur un petit nombre de femelles, que des lapines dont on tuait les petits dès la naissance pouvaient accomplir une série de gestations successives sans diminution de fertilité et de prolificité ni altération de leur condition physique. Nos premiers travaux (PRUd'hon et BEI, I968; Sinse; I968) ont indiqué que les lapines pouvaient donner naissance à des portées de taille normale après accouplement le lendemain de la parturition, même lorsqu'elles allaitent. Cependant, les observations de Hammond et Marshall (I925) laisseraient penser que ce n'est pas toujours le cas. De même, Adams (I967) a constaté qu'il existait une incompatibilité entre gestation et allaitement lorsque les femelles, saillies peu après la mise bas, avaient un faible niveau nutritionnel; les femelles bien nourries, au contraire étaient capables de mener à terme leur gestation. HAFEz et Ishibashi (I965) ont observé que des lapines primipares accouplées à nouveau après I5 jours d'allaitement avaient un nombre de blastocystes viables plus faible que des lapines de même âge en première gestation, ou des primipares n'ayant pas allaité au cours des quinze jours précédant la saillie.

La définition d'un rythme optimum de reproduction des lapines nécessite donc une meilleure connaissance des facteurs qui peuvent agir sur leur fertilité et leur prolificité et, plus spécialement, des relations existant entre l'allaitement et la gestation.

Nous donnons ici les premiers résultats d'études portant sur la recherche d'un rythme optimum de reproduction chez les lapines. Ils concernent plus particulièrement les rapports entre la durée de l'intervalle parturition-saillie et les performances de reproduction des lapines au niveau d'une gestation et d'une portée. 


\section{MATÉRIEL ANIMAL FT MÉTHODES EXPÉRIMENTALES}

Ces études ont été conduites conjointement au Laboratoire de Zootechnie de l'E. N.S. A. de Montpellier (essai I) et à la station de Génétique quantitative et appliquée à Jouy-en-Josas (essai 2).

Essai r.

D'octobre I966 à avril I968, 70 lapines “communes " issues de l'élevage du Laboratoire de Zootechnie de 1'E. N. S. A. de Montpellier ont été soumises, de façon échelonnée, à des tentatives systématiques d'accouplement dès le lendemain de la mise bas, ces tentatives étant répétées chaque jour jusqu'à l'obtention d'une saillie effective. Les conditions d'élevage et d'alimentation de ces femelles ainsi que les techniques de sevrage des lapereaux, pratiqué à I 4 ou 2 I jours, ont déjà été décrites (Prud'Hon, I967; BEL et PRUD'HON, I968).

Les gestations étaient contrôlées par palpation I 4 jours après la saillie ou constatées au moment de la mise bas présumée.

Les données ont été groupées en fonction de l'intervalle parturition-saillie, ce qui nous a conduit à constituer 3 lots:

$$
\begin{aligned}
& \text { lot I : intervalle compris entre o et } 6 \text { jours (moyenne I, } 6 \text { jour) } \\
& \text { lot } 2 \text { : intervalle compris entre Io et } 20 \text { jours (moyenne I 5, I jours) } \\
& \text { lot } 3 \text { : intervalle supérieur à } 30 \text { jours (moyenne } 37,6 \text { jours). }
\end{aligned}
$$

Le lot 2 comprend des femelles ayant eu un accouplement différé ainsi que des femelles détectées vides à la palpation et saillies à nouveau.

Le lot 3 comprend essentiellement des femelles saillies Io à 20 jours après parturition, détectées vides à la palpation $I_{4}$ jours plus tard et saillies à nouveau.

Les lapines étaient pesées dans les 24 heures suivant la parturition et après chaque saillie. Les lapereaux étaient sexés, pesés et identifiés le jour de la naissance selon la méthode décrite par Bel et CANtrer (1968). Le nombre des petits allaités était contrôlé au moment des pesées hebdomadaires.

\section{Essai 2.}

De juillet I967 à juin I968, 66 lapines pour la plupart (5I) de race Argenté de Champagne, ont été soumises à deux rythmes théoriques de reproduction, les lapereaux étant sevrés à trois âges. Ces lapines étaient réparties en six lots d'effectifs comparables correspondant aux six combinaisons factorielles de deux dates de présentation au mâle après la mise bas : un jour ou neuf-dix jours, et de trois âges de sevrage des lapereaux : I 6, 2 I ou 28 jours. Un lot considéré comme témoin correspondait à des lapines présentécs au mâle à partir du $24^{\text {c }}$ jour après la mise bas, les lapereaux étant sevrés à 28 jours.

Les lapines étaient accouplées à quelques instants d'intervalle à un mâle Argenté de Champagne et à un mâle Fauve de Bourgogne en vue d'assurer le même milieu maternel aux descendants de race pure ou croisés.

Les lapines étaient élevées dans des cages entièrement grillagées à l'intérieur d'un bâtiment fermé dont la ventilation était statique ; elles disposaient d'une boîte à nid placée avant la parturition et restant en place pendant tout le séjour des jeunes avec la mèrc. Les cages étaientéquipées d'un système d'abreuvement automatique par clapet. Pendant les 7 premicrs mois de l'expérimentation, les lapines allaitantes ont reçu à volonté un granulé dosant, 22,4 p. Ioo de matière azotée totale et i 2 p. Ioo de cellulose brute. Finsuite, la moitié du cheptel, répartie également dans les 7 catégories expérimentales, a reçu, toujours à volonté, un granulé dosant I 4 p. Ioo de cellulose brute et seulement $I_{4}, 5 \mathrm{p}$. roo de matière azotée totale. L'autre moitié recevait toujours l'aliment précédent.

Les lapines étaient présentées au mâle à la date prévue puis tous les jours successifs à partir de cette date, jusqu'à ce qu'il y ait accouplement. Le điagnostic de la gestation était effectué par palpation de la lapine io jours après la saillie, les lapines présumées non gestantes étant présentées immédiatement au mâle. Nous avions constitué 3 groupes expérimentaux de lapines suivant les dates de saillies théoriques de un, neuf-dix, vingt quatre jours après la parturition. Dans chacun de ces groupes, il y eut un étalement des saillies effectuées qui nous a conduits à grouper les données en quatre lots en fonction de l'intervalle réel parturition-saillie suivante:

$$
\begin{array}{llrr}
\text { lot } 1: \text { intervalle compris entre } & \text { o et } 7 \text { jours } \\
\text { lot } 2: & - & 9 \text { et } 17 \text { jours } \\
\text { lot } 3: \text { - } & - & 20 \text { et } 28 \text { jours } \\
\text { lot } 4: \text { intervalle supéricur à } 30 \text { jours. }
\end{array}
$$


La plupart des données sont groupées respectivement à un jour (69 saillies sur les 87 du lot $\mathrm{r}$ ), de 9 à $\mathbf{2} 2$ jours ( 98 saillies sur les I I 9 du lot 2 ) et de 2 I à 26 jours ( 44 saillies sur les 53 du lot 3 ). Le lot 2 comprend les saillies des lapines présentées au mâle 9 -Io jours seulement après la mise bas (82 saillies sur I 19) ainsi que celles de quelques lapines détectées vides par palpation à la suite d'une saillie post-partum ( 37 sur 1 r 9 ). De même, le lot 3 comprend les saillies des lapines du lot témoin, présentées au mâle 24 jours seulement après la mise bas ( 25 saillies) et les saillies des femelles détectées non gestantes à la suite de saillies plus proches de la mise bas ( 28 saillies).

Dans les lots I et 2 l'allaitement durait jusqu'aux $16^{\mathrm{e}}, 2 \mathrm{I}^{\mathrm{e}}$ ou $28^{\mathrm{e}}$ jours après la mise bas, sauf s'il y avait disparition complète de la portée par mortalité. Dans les lots 3 et 4 l'allaitement durait, dans la plupart des cas, jusqu'au $4^{2} \mathrm{e}$ jour après la mise bas, sauf pour quelques lapines dont la saillie était théoriquement prévue à I jour ou 9-Io jours après la parturition et qui ont pris du retard, ces saillies n'ayant pas été fécondantes.

\section{Calculs réalisés.}

L'analyse a d'abord porté sur la comparaison des moyennes des 3 lots (essai r) ou des 4 lots (essai 2) pour les variables : taux de gestation, nombre de lapereaux nés vivants, nés "total ", et durée de gestation (essai I).

Le taux de gestation a été apprécié par le pourcentage de femelles saillies, détectées gestantes au moment de la palpation (essai 2) et par le pourcentage de femelles saillies mettant bas (essai $\mathbf{I}$ ), la palpation n'ayant pas été systématiquement pratiquée dans cet essai.

Les tests de comparaison ont été faits à la suite du calcul du $\chi^{2}$ de Pearson en ce qui concerne, les taux de gestation, et du $\mathrm{F}$ de Snedecor pour les effectifs et durées de gestation.

Les mêmes calculs ont été réalisés à l'intérieur de chaque lot, pour tester la signification des effets des facteurs suivants sur les mêmes variables (durée de gestation exclue) :

- effet du numéro de portée,

- effet de l'effectif de portée au moment de la parturition sur les résultats de la saillie suivante,

- effet de l'effectif allaité jusqu'à I 4 jours sur les résultats des saillies survenant au cours de cet allaitement.

Nous avons également testé à titre indicatif et dans l'essai I seulement :

- l'effet de l'époque de saillie,

- l'effet des variations de poids des lapines au cours d'une gestation sur les résultats de la saillie suivante.

\section{RÉSULTATS}

\section{Résultats généraux}

Dans le tableau I nous indiquons les résultats de comparaison globale des différents lots pour chacun des deux essais. Nous avons fait figurer à côté des moyennes leurs écarts-types.

\section{a) Taux de gestation.}

La différence de taux de gestation entre les divers lots est hautement significative dans les deux essais : ces taux sont nettement plus élevés lors de la saillie, ro à 20 jours après la mise bas que lors de celle à $0-7$ jours (respectivement 85 et 82 p. Ioo contre 56 et 45 p. Ioo, dans les deux essais). Ce taux redevient plus faible dans le lot 3 de l'essai I et surtout le lot 4 de l'essai 2 . On trouve dans ces deux lots des lapines de faible fertilité.

Dans le lot 2 de l'essai 2 , nous avons également calculé séparément les taux de gestation pour les lapines dont la date de saillie théorique était 9 -Io jours et les autres. Ils sont respectivement de $8 \mathrm{I}, 7$ p. Ioo et $8 \mathrm{I}, \mathrm{I}$ p. Ioo. Cette absence de différence justifie le regroupement des deux catégories de résultats dans le lot 2. Dans le lot 3 , les lapines considérées comme témoins ont un taux de gestation de 84,0 p. Ioo et les autres de 6o,6 p. Ioo. La différence n'est pas significative au seuil 5 p. Ioo, 


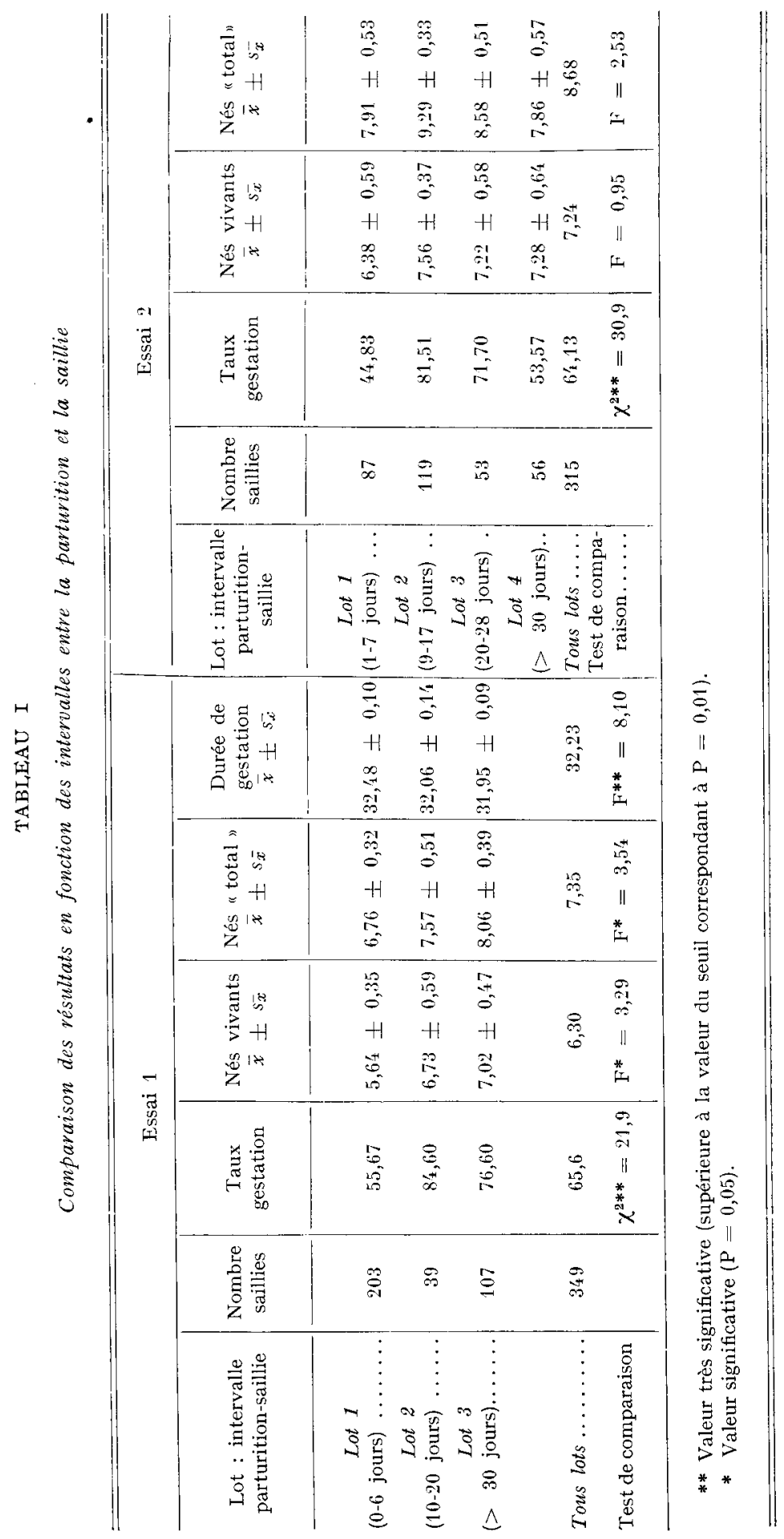


mais elle correspond à une probabilité située entre 5 et Io p. roo. Il y aurait donc un facteur individuel, les lapines non fécondées lors de la saillie 9 -I 7 jours après la parturition ayant tendance à être moins fertiles. Cet effet propre à l'animal ne se manifeste pas chez les lapines non fécondées lors de la saillie à I-7 jours et waillies à nouveau à 9-I7 jours. Cela semble indiquer que dans ce cas c'est la date de saillie qui est le facteur déterminant et qu'il masque les effets individuels.

\section{b) Prolificité.}

Dans le lot I on note une tendance des lapines à donner naissance à des portées d'effectif plus faible que dans les autres lots : dans les deux essais, le nombre de lapereaux nés vivants par portée est inférieur d'un peu plus d'un individu dans le lot I à ce qu'il est dans le lot 2. Cette différence, significative dans l'essai I, ne l'est pas dans l'essai 2. Le nombre total de lapereaux nés par portée suit une évolution analogue. Les effectifs sont, ici aussi, plus faibles dans le lot I ; les différences sont significatives $(\mathrm{P}<\mathrm{O}, \mathrm{OI})$ dans l'essai $\mathrm{I}$ et proche du sauil de signification $(\mathrm{P}=0,05)$ dans l'essai 2.

Dans le lot 2 de l'essai 2, nous n'avons pas de différence significative entre la prolificité des lapines dont la date théorique de saillie était à 9 -Io jours après la parturition, et les autres. Il en est de même entre les deux groupes de lapines du lot 3 de l'essai 2 .

\section{c) Durée de gestation.}

Les saillies ayant lieu à heure fixe en début d'après-midi et les mises bas étant constatées également à la même heure, le calcul d'une durée de gestation est relativement imprécis. Cette réserve faite, nous avons constaté (essai I) une durée moyenne de gestation des lapines du lot I supérieure d'une demi-journée à celles constatées dans les autres lots.

Cependant à effectif de portée égal, la durée de gestation ne différait pas sensiblement d'un lot à l'autre.

Il semble par conséquent que cette différence soit liée principalement à la naissance, dans ce lot $I$, d'une plus forte proportion de portées de très faible effectif ( I à 3 petits). Quel que soit le lot, en effet, la durée de gestation pour des portées de taille réduite excède en général de I à 3 jours la durée normale, ce qui est en accord avec les observations de VAïDA (I962).

\section{d) Comportement des lapines après parturition.}

- Essai 1 . tion.

Les lapines étaient présentées à l'accouplement tous les jours après la parturi-

Quarante-trois lapines ont toujours accepté l'accouplement au cours des six premiers jours suivant la mise bas.

Vingt lapines ont eu, une fois au moins, un accouplement différé de Io à 20 jours, quatre d'entre elles ont eu 2 accouplements différés et une lapine a eu 3 accouplements différés (sur 8). Cependant aucune de ces lapines n'a systématiquement refusé l'accouplement après chaque parturition. 
Signalons également dans cet essai que trois lapines ont attendu plus de 30 jours avant d'accepter l'accouplement. Elles ont été éliminées ce qui ne permet pas de savoir si cette anomalie eût été reproductible.

- Essai 2 .

I a régularité des femelles vis-à-vis du rythme de reproduction n’a pas été étudiée, l'expérience étant trop limitée dans le temps. Cependant, il n'existe pas de différences significatives entre lapines pour l'intervalle parturition-saillie fécondante, l'analyse statistique ayant été faite pour chaque groupe expérimental de lapines suivant les dates de saillie théoriques.

\section{Facteurs de variation}

a) Age au seirage.

L'influence éventuelle de 1'âge au sevrage peut être étudiée clans l'essai 2 . Il n'y a pas d'effet significatif sur le taux de gestation dans les lots I et 2. D'ailleurs, dans le lot I, la gestation était détectée par palpation abdominale Io jours après la saillie, donc avant le prenier sevrage possible ( 6 jours), et nous n'avons pas observé de cas de lapines détectées gestantes qui n'auraient pas mis bas. De même, il n'apparaît pas d'influence significative de l'âge au sevrage sur la prolificité à la portée suivante.

b) Effectif né et effectif allaité jusqu'à 14 jours.

Sur les tableaux 2 et 3 nous avons reporté respectivement les valeurs du taux de gestation et du taux de prolificité des lapines à la suite de la $\mathbf{n}^{\mathrm{ieme}}$ saillie, en fonc-

TABILAU 2

I'aleurs du taux de gestation à la saillie de rang $n$ en fonction des classes d'effectifs de iapercaux nés et allaités jusqu'à 14 jours dans la portée de rang $n-1$

\begin{tabular}{|c|c|c|c|c|c|c|c|c|}
\hline \multirow[b]{3}{*}{ Portée $n$} & \multicolumn{4}{|c|}{ Lot 1} & \multicolumn{4}{|c|}{ Lot 2} \\
\hline & \multicolumn{2}{|c|}{ Essai 1} & \multicolumn{2}{|c|}{ Jissai 는 } & \multicolumn{2}{|c|}{ Essai 1} & \multicolumn{2}{|c|}{ Essai ? } \\
\hline & $\begin{array}{l}\text { Nombre } \\
\text { saillies }\end{array}$ & $\mid \begin{array}{c}\text { Taux ges- } \\
\text { tation } \\
(\%)\end{array}$ & $\begin{array}{l}\text { Nonubre } \\
\text { saillies }\end{array}$ & $\begin{array}{c}\text { Taux ges- } \\
\text { tation } \\
(\%)\end{array}$ & $\begin{array}{c}\text { Nombre } \\
\text { saillies }\end{array}$ & $\mid \begin{array}{c}\text { Taux ges- } \\
\text { tation } \\
(\%)\end{array}$ & $\begin{array}{c}\text { Nombre } \\
\text { saillies }\end{array}$ & $\begin{array}{c}\text { Taux ges } \\
\text { tation } \\
(\%)\end{array}$ \\
\hline Effertif no 1 a & $3 i$ & 50,0 & 19 & $4 \bar{Z}, 4$ & $i_{i}$ & $x: 3,3$ & 18 & 83,3 \\
\hline Effectut né\{'tà $6 \ldots . .\}$. & $5 t$ & 53,7 & 25 & 60,0 & 11 & 90,9 & 29 & 86,2 \\
\hline 7 à $9 \ldots \ldots$ & 62 & 50,0 & 29 & 34,5 & 14 & 87,5 & 36 & 72,2 \\
\hline Fortee $n-1\}>9 \ldots$ & 53 & 67,9 & 14 & 35,7 & 8 & 75,0 & 36 & 80,6 \\
\hline Test de comparaison & & NS & & NS & & NS & & $x \mathrm{~S}$ \\
\hline Jà 30 à. & 75 & 48,0 & $1 \pm$ & 50,0 & 17 & $8:, 3$ & 24 & 83,3 \\
\hline Liffectif $\quad\left\{\begin{array}{lll}x_{1} & \text { à } & 6\end{array} \ldots\right.$ & 72 & 51,4 & 14 & 56,0 & 16 & 87,5 & 30 & 83,3 \\
\hline allaité $\quad(7$ à $9 \ldots \ldots$ & 47 & 61,7 & 10 & 35,7 & 6) & 83,3 & 魚 & 75,0 \\
\hline Portee $n-1\{>9 \ldots$ & $1:$ & 83,3 & 3 & 30,0 & & & 21 & 81,0 \\
\hline Test de comparaison & & NS & & NS & & NS & & NS \\
\hline & & & & 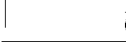 & $i$ & & & \\
\hline
\end{tabular}


M. PRUD'HON, R. ROUVIER, J. CAEL, L. BEL,

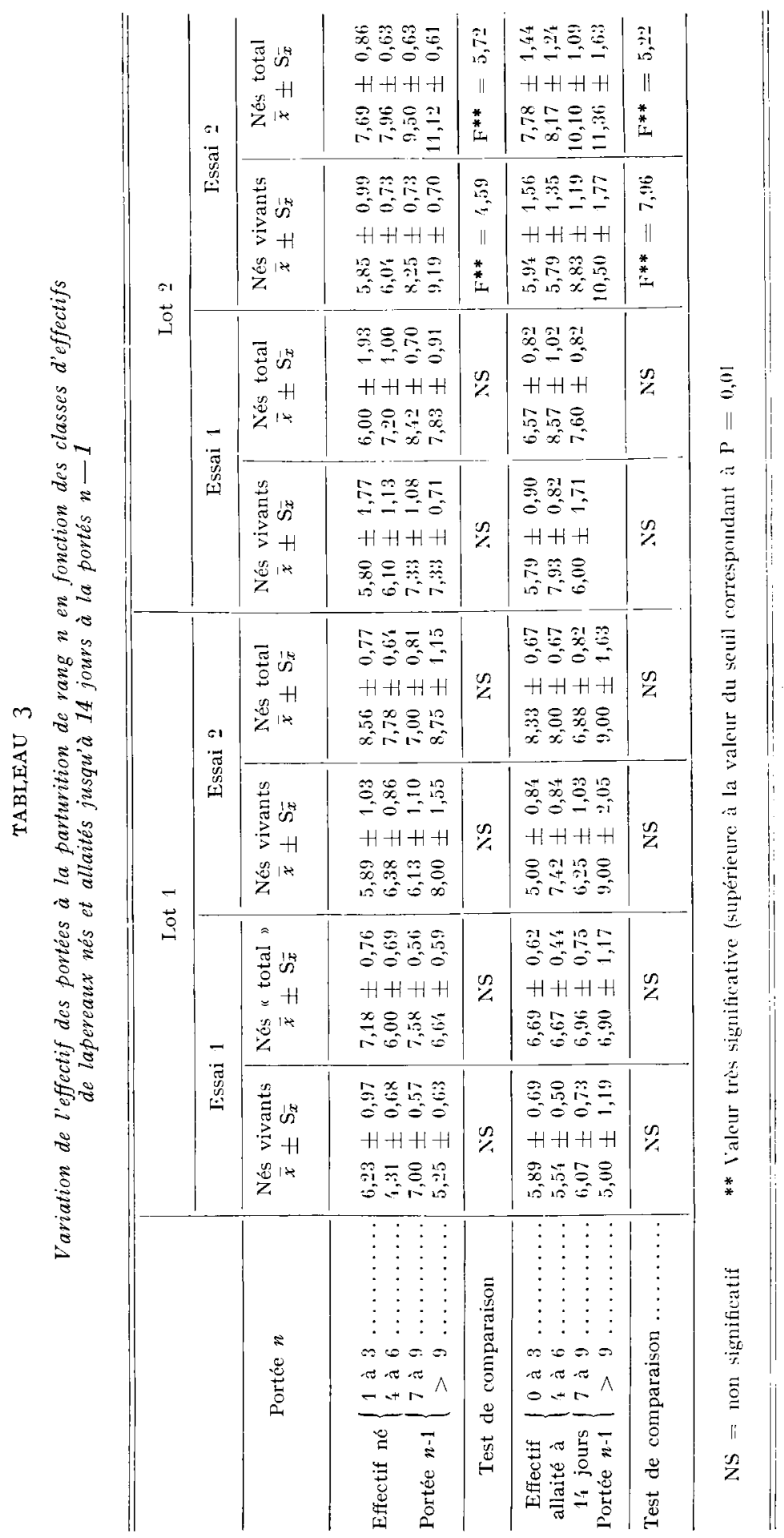


tion du nombre de lapereaux nés ou allaités pendant I 4 jours après la $n$-I ieme parturition.

Quel que soit le lot ou l'essai, le taux de gestation ne dépend pas significativement de ces deux facteurs.

Le taux de prolificité, dans chaque lot 2, a tendance, par contre, à varier dans le même sens que le nombre de lapereaux nés ou allaités jusqu'à I 4 jours à la précédente portée. Les différences enregistrées sont hautement significatives dans l'essai 2 , non significatives dans l'essai I où peu de données sont disponibles.

On ne retrouve pas ce facteur individuel de reproductibilité des performances dans le lot I de chacun des essais. Il sera intéressant de préciser à partir d'observations plus nombreuses si ce fait se confirme; il semble, en effet, indiquer un léger antagonisme entre le nombre de lapereaux nés et allaités à une portée et le nombre de lapereaux nés à la portée suivante lorsque la saillie post-partum est fécondante. Cet antagonisme éventuel ne se manifesterait plus, chez les lapines que nous avons étudiées s'il existe un intervalle de Io à 20 jours entre la parturition et la saillie fécondante.

\section{c) Numéro de portée.}

L'influence du numéro de portée sur le taux de gestation et la prolificité des lapines semble faible, dans le cadre de ces essais où nous ne considérons que les numéros de portée égaux ou supérieurs à deux, et où il y a relativement peu de portées de rang élevé. Cependant, en ce qui concerne le lot I, nous avons enregistré dans les deux essais une différence significative dans le nombre des lapereaux nés en fonction de ce facteur de classification. Il semble qu'il y ait dans ce lot une variation en dents de scie des effectifs nés vivants et nés " total", des portées d'effectif plus faible succédant à des portées d'effectif important. Ce résultat devrait cependant être confirmé sur des données plus nombreuses pour mériter une discussion.

Dans les lots 2,3 et 4 aucun test concernant l'influence du numéro de portée n'est significatif. L'effet numéro de portée ne nous apparaît donc pas suffisamment important pour avoir perturbé notre comparaison globale des lots (tabl. I) où tous les numéros de portée sont regroupés.

\section{d) Époque de saillie.}

Nous n'avons pas mis en évidence d'influence notable de l'époque de saillie, aucun des tests n'ayant été significatifs. Il faut cependant signaler que dans l'essai I, dans tous les lots, le nombre de lapereaux nés avait tendance à être plus élevé au cours du premier semestre, ce qui est conforme aux constatations de nombreux auteurs (Strtman et al., I964; Hammond, I965).

\section{e) Variations pondérales des lapines.}

Aucun des tests de comparaison n'est significatif. Il semble cependant, dans l'essai I, qu'il y ait une tendance à l'abaissement du taux de gestation lorsque les femelles ayant perdu beaucoup de poids au cours de la précédente gestation sont saillies au cours des six premiers jours suivant la parturition. 


\section{DISCUSSION}

Il apparaît une concordance certaine entre les résultats des deux essais : la fertilité et la prolificité des lapines saillies immédiatement après la mise bas est plus faible que lors d'une saillie plus tardive. Un délai de ıo jours est suffisant pour rétablir un niveau normal de fécondité. L'intervalle parturition-saillie est de loin le plus important des différents facteurs de variation étudiés tant pour le taux de gestation que pour la prolificité. Les autres facteurs de variation n'apparaissent en général pas significatifs dans cette expérimentation, ce qui confirme le rôle prépondérant de la date de saillie devant l'effectif né ou allaité. Ainsi, l'antagonisme lactationgestation ne semble se manifester, dans ces essais, qu'à la suite de saillies post-partum.

Les raisons d'une réduction de fertilité et de prolificité chez les seules lapines allaitantes saillies post-partum n'apparaissent pas clairement. HAFEZ et RAJAKOSKI (I964) ont montré que le taux d'ovulation et la réponse à des traitements induisant la superovulation étaient analogues chez les primipares saillies post-partum et les nullipares. HAFrz et Ishibashi (I965) ont confirmé ces résultats. On pourrait donc exclure l'hypothèse d'un abaissement du taux d'ovulation chez les lapines après le part. Nous avons d'ailleurs enregistré après de telles saillies, la naissance de portées de I $_{5}$ et I7 petits. HAFiz et Ishibashi (I965) ont, par contre, constaté chez des lapines de race Néo-Zélandaise blanche que le nombre de blastocystes viables récupérés 6 jours après accouplement était plus faible chez les lapines saillies I 5 jours après parturition et allaitantes que chez les primipares saillies post-partum dont on abattait les petits à la naissance et chez les nullipares ; cela malgré la présence d'un nombre de corps jaunes sensiblement égal. Cette constatation ne s'accorde pas avec nos résultats, les lapines saillies Io-20 jours après la parturition ayant des meilleures performances de fertilité et de prolificité.

L’influence néfaste de la lactation sur le maintien de la gestation semble bien établie seulement lorsque la saillie a lieu peu de temps après la parturition : HAmmond et Marshal. (I925) ont constaté que les embryons dégénéraient au stade blastocyste lorsque le nombre de petits qui tètent est supérieur à 3 ou 4 . Adams (I967) a confirmé l'existence d'une relation entre l'interruption de la gestation et l'effectif allaité, cependant le seuil critique serait plus ou moins élevé selon que le niveau d'alimentation des lapines est lui-même plus ou moins élevé. Ainsi, pourrait-on expliquer que dans nos essais, où les femelles disposaient à volonté, et comme seul aliment, d'un granulé riche en protéines nous n'ayons pas enregistré de différences significatives du taux de gestation en fonction du nombre de petits nés précédemment et allaités par les lapines saillies post-partum. Deux phénomènes toutefois permettent de penser que dans ces essais il y a un léger antagonisme entre le nombre de lapereaux nés et allaités à une portée et le nombre de lapereaux nés à la portée suivante, à la suite d'une saillie post-partum:

- les effectifs de portées successives ont tendance à varier en dents de scie;

- les performances des femelles saillies après le part sont peu reproductibles, ce qui contraste avec la reproductibilité constatée dans le cas de saillies survenant Io à 20 jours après celui-ci. Mais ces observations demandent confirmation. 
Le maintien de la gestation et la prolificité des lapines en lactation dépend aussi, sans doute, de leur état physiologique au moment de l'accouplement. Les femelles ayant perdu beaucoup de poids au cours d'une gestation ont une faible fertilité après saillie post-partum (essai I) ; par contre, les femelles saillies Io à 20 jours après la mise bas, qui, malgré leur lactation, atteignent leur poids maximum au moment de l'accouplement (PRUD'HON, I967), ont un taux de gestation et un taux de prolificité élevés. Chez ces femelles, le taux de gestation est indépendant de l'effectif né ou allaité à la portée précédente et le taux de prolificité est très reproductible, les lapines les plus prolifiques à la portée $n$ - I donnant des portées d'effectif élevé à la portée $n$.

L'antagonisme gestation-allaitement, observé après les saillies post-partum, résulte vraisemblablement d'une modification de l'équilibre endocrinien régissant le maintien et le fonctionnement du corps jaune, la préparation de l'utérus à la nidation ou les deux à la fois. Chez la Lapine, le maintien et le fonctionnement du corps jaune dépendent directement des oestrogènes (revue de DESCI,IN, I954; KEYES et Nalbandov, 1967). Cependant, selon Sires et al. (I968), FSH en agissant sur la croissance des follicules, et la prolactine sur le tissu interstitiel, seraient essentielles pour la sécrétion d'œestrogènes. Ces hormones pourraient donc avoir une action indirecte sur le corps jaune. ADAms (1967) a constaté qu'un traitement à base de préparations antehypophysaires de cheval permettait d'éviter la régression des corps jaunes et la réduction du nombre des fotus, observables du $6^{\mathrm{e}}$ au $\mathrm{I}^{\mathrm{e}}$ jour suivant la saillie post-partum, chez les lapines allaitantes maintenues à un bas niveau alimentaire. I1 estime que l'allaitement entraîne une production suboptimale de FSH.

En ce qui concerne la nidation, elle est surtout sous le contrôle de la progestérone (revue de MAYkr, I963). En utilisant la technique du transfert d'embryons, SmidT, THOMSEN et HAFEZ (I968) ont constaté que la lactation avait un effet néfaste sur l'implantation des embryons et qu'il était possible d'atténuer sensiblement cet effet par l'injection quotidienne de progestérone. Ainsi, la baisse de fertilité et de prolificité que nous avons observée à la suite des saillies post-partum pourrait être due à une moins bonne nidation liée à l'existence d'une lactation simultanée. Mais pourquoi cela ne se produirait-il plus lors des saillies réalisées Io à 20 jours après parturition, c'est-à-dire à l'époque où la production laitière de la Lapine passe par un maximum?

On peut aussi penser que cette moins bonne nidation pourrait être liée à un état particulier de l'utérus après la mise bas.

\section{CONCLUSION}

En conclusion, ces résultats posent, au niveau de leur explication biologique, un certain nombre de problèmes qu'il serait utile d'étudier du fait de leur intérêt en relation avec les possibilités d'application pratique en élevage. A ce point de vue, ces essais ont montré qu'avec le type d'alimentation que nous avons utilisé il était possible d'obtenir des résultats satisfaisants en faisant saillir les lapines dès la parturition et en accouplant à nouveau les lapines non gestantes à partir du Io jour après cette saillie. Les résultats étaient également satisfaisants en faisant saillir des lapines IO-I2 jours après la parturition. Dans ce cas des taux de gestation et de prolificité nettement plus élevés permettaient de compenser le retard de 1'accouplement. Malgré 
ces premières indications, ces observations sont insuffisantes pour nous permettre de définir le rythme de reproduction optimum pour les lapines destinées à la production intensive de lapereaux. Des expérimentations complémentaires portant sur un nombre plus important de lapines et visant à étıdier leur longévité, ainsi que sur diverses races, semblent nécessaires. Pour un intervalle parturition-saillie donné, les taux de gestation et la prolificité peuvent en effet être aussi fonction de la race et de la souche. Cette expérimentation sur plusieurs races ou souches, en permettant d'analyser complètement la variabilité des résultats, leur donnerait plus de généralité et d'intérêt et favoriserait très probablement la recherche d'explications biologiques.

Rę̧u pour publication en Juillet 1969.

\section{REMERCIEMENTS}

Nous remercions Mme Suzanne Torres et M. F. Lebas pour leurs lectures critiques du manuscrit et leurs suggestions.

\section{SUMMARY}

\section{ACTION OF THE BIRTH-MATING INTERVAL ON THE FERTILITY AND PROLIFICACY OF RABBITS}

Two trials were performed in 2 different matings to study the influence of the birth-mating interval on rabbit fertility and prolificacy.

In Trial I female rabbits of the commune breed were presented for mating the day their young were born, and those not pregnant were remated i 5-35 days later.

In Trial 2 Argenté de Champagne female rabbits were presented for mating $\mathrm{I}$, 10, or 24 days after the young were born.

The results were analyzed taking into account the birth-mating interval. The interval values are the following :

\begin{tabular}{ll}
\multicolumn{1}{c}{ Trial I } & \multicolumn{1}{c}{ Trial 2} \\
Lot I : 0-6 days & Lot I : $1-7$ days \\
Lot $2:$ I0-20 days & Lot $2: 9-17$ days \\
Lto $3:$ more than 30 days & Lot $3: 20-27$ days \\
& Lot $4:$ more than 30 days
\end{tabular}

In the 2 trials, the pregnancy rates (number of births for Ioo matings) are significantly lower in Lot $\mathrm{I}$ than in Lot 2, the respective values being 56 and $45 \mathrm{p}$. Ioo instead of $85 \mathrm{p}$ and $82 \mathrm{p}$. Ioo. The average number of young born per litter is lower (about I-I.5 young) in Lot $I$ than in the other Lots. This difference is significant in Trial $\mathrm{I}$.

We have studied the relationship between the number of young resulting from the $n$ - I birth suckled for $\mathrm{r}_{4}$ days, and the results of the $\mathrm{n}^{t h}$ mating.

There is no evidence of antagonism between suckling and pregnancy when mating occurs Io-20 days after birth.

The different factors having a negative action on the results of post-partum mating are discussed. 


\section{RÉFÉRENCES BIBLIOGRAPHIQUES}

Adams C. E., 1967. Concurrent lactation and pregnancy in the rabbit. J. Reprod. Fert., 13, 35 I-352. Bel L., Cantier J., I968. Sexez et identifiez vos lapereaux dès la naissance Revne Élev. I968, n 6 et 7, I33-139.

Ber. L., Prud'hon M., r968. Perspectives d'intensification de la production du lapin de chair, grâce au sevrage précoce des lapereaux et à la reproduction continue des lapines. Bull.tech. Inf. Ingrs. Servs. agric. I968, 229, 387-393.

CASADY R. B., I965. Onze portées par an. Vos lapins., 85, 14-20.

Desclin L., I953. Les facteurs qui déterminent la longueur de vie des corps jaunes et conditionnent leur activité fonctionnelle. II Réunion des Endocrinologistes de langue française., 1, 32. Masson, Paris.

Guthrie D., 1966. "Creep feeding," sevrage précoce et accélération du rythme reproductif. Vos lapins., 86, $18-24$.

Hafez E. S. E., Rajakoski E., I964. Growth and survival of blastocysts in the domestic rabbit. I. - Effect of maternal factors J.Reprod. Fert., $7,229-249$.

HAFEz E. S. E., Ishibashi I., 1965. Effect of lactation and age at first breeding on size and survival of rabbit blastocysts. Int. J. Fert., 10, 47-55.

Hammond J., Marshall F. M. A., 1925. Reproduction in the Rabbit. Edinburgh, I925, cité par AsDell S. A. I946. Patterns of mammalian reproduction. Comstock New York.

HАммоN J., I965. The effects of high and low planes of nutrition on reproduction in rabbits. N.Z. $J$. agric. Res., 8, 708-717.

Keyes P. L., Natbandov A. V., I967. Maintenance and function of corpora lutea in rabbits depend on estrogen. Endocrinology., 80, 938-946.

Mayer G., 1963, in. Enders A.C., Delayed implantation. 2I3-23I. Univ. Chicago press.

Prud'ron M., 1967. L'appétit du lapin alimenté à sec. Bull. tech. Inf. Ingrs. Servs. agric. 1967., 219, $383-398$

PRUD'hox M., Bel L., I968. Le sevrage précoce des lapereaux et la reproduction des lapines. $-A n n$. Zootech., 17, 23-30.

Sinse-CAEL J., I968. Étude des possibilités d'utilisation zootechnique du rythme de reproduction accéléré des lapines et du sevrage précoce des lapereaux. (Communication personnelle.)

Sitrman D. B., Rollins W. C., Sittmay K., Casady R. B., r964. Seasonal variation in reproductive traits of New Zealand White rabbits. J. Reprod. Fert., 8, 29-37.

Smidt D., Thomsen J. J., Hafez E. S. E., I968. Untersuchungen zum emfluh der laktation auf implantation und fotalentwicklung beim kaninchen. Z. Tierziicht Ziucht Biol., 84, I I5-126.

Spies H. G., Hilliard, J., SAWYER C, H., I968. Maintenance of corporalutea and pregnancy in hypophysectomized rabbits. Endocrinology., 83, 354-366.

VAï̄a M., 1962. Duration of pregnancy in rabbits. Wien tierärzt Mschr., 49, 33I-332 in. Anim. Breed. Abstr. I963., 31, no 604 . 